\title{
The euglobulin lysis time test: An ineffectual monitor of the therapeutic inhibition of fibrinolysis
}

\author{
J. KATZ, A. LURIE, D. BECKER, AND J. METZ \\ From the Department of Haematology, South African Institute for Medical Research, Johannesburg
}

SYNOPSIS In two clinical situations associated with hyperfibrinolysis the administration of $\vec{\oplus}$ antifibrinolytic drugs resulted in clinical haemostasis. The dilute clot lysis time and fibrin $\frac{\mathbb{P}}{\overparen{T}}$ plate activity test but not the euglobulin lysis time reflected this control of excessive fibrinolysis $\stackrel{\mathbb{1}}{3}$ by the antifibrinolytic drugs. The inhibition by epsilon-amino-caproic acid (EACA) of hyper-黑 fibrinolysis induced in vitro was reflected by the dilute clot lysis time but not by the euglobulin $\overrightarrow{0}$ lysis time. Paper chromatography of the supernatant and euglobulin fractions as prepared for the euglobulin lysis time test from plasma with added EACA demonstrated that some $85 \%$

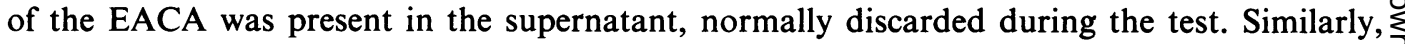
cellulose-acetate electrophoresis of the supernatant and euglobulin fractions from plasma containing Trasylol demonstrated the drug in the supernatant only. These findings indicate $\frac{\mathscr{Q}}{\nabla}$ that when acetic acid is added to plasma containing EACA or Trasylol only a small proportion $\stackrel{\varrho}{\Rightarrow}$ of the drug is precipitated with the euglobulin fraction. The euglobulin lysis time is thus an inaccurate index of the neutralization of hyperfibrinolysis by antifibrinolytic drugs.

Hyperfibrinolysis has been associated with a variety of disease entities, occurring either secondary to disseminated intravascular coagulation (MacKay, 1965) or more rarely as a primary pathological entity (Merskey, Johnson, Kleiner, and Wohl, 1967). The therapeutic agents most frequently used to control this excessive fibrinolysis are the amino-acid derivatives, EACA and tranexamic acid, and the kallikrein inhibitor, Trasylol. Laboratory tests for measuring fibrinolytic activity include the dilute clot lysis time, the euglobulin lysis time, the fibrin plate activity test, tests for fibrinogen degeneration products, and the plasminogen assay. By virtue of its ease of performance the euglobulin lysis time has been used by Pechet, Groth, and Daloze (1969) to monitor therapy with antifibrinolytic drugs. The present report, however, indicates that this test fails to reflect changes in fibrinolysis produced by antifibrinolytic drugs. The reason for this is that only a small amount of the antifibrinolytic drugs is precipitated with the euglobulin fraction, the major portion being discarded in the supernatant during the performance of the test.

\section{Clinical Material}

Two clinical situations were studied: (1) a patient with acute promyelocytic leukaemia and increased fibrinolysis as evidenced by shortened euglobulin and dilute clot lysis time, lysis on a fibrin plate, and low levels of plasminogen and fibrinogen: $>$ (2) anhepatic baboons undergoing experimental xenograft liver transplants. Removal of then baboon liver is associated with a rapid increase in fibrinolytic activity, with shortened euglobulin 0 and dilute clot lysis times and low levels of $\tilde{\omega}$ plasminogen.

\section{Methods}

\section{FIBRINOLYTIC TESTS}

Venous blood was collected into $3.8 \%$ trisodium citrate in a ratio of 9:1. The euglobulin lysis time (Nilsson and Olow, 1962), dilute clot lysis timeo Received for publication 23 December 1969. 

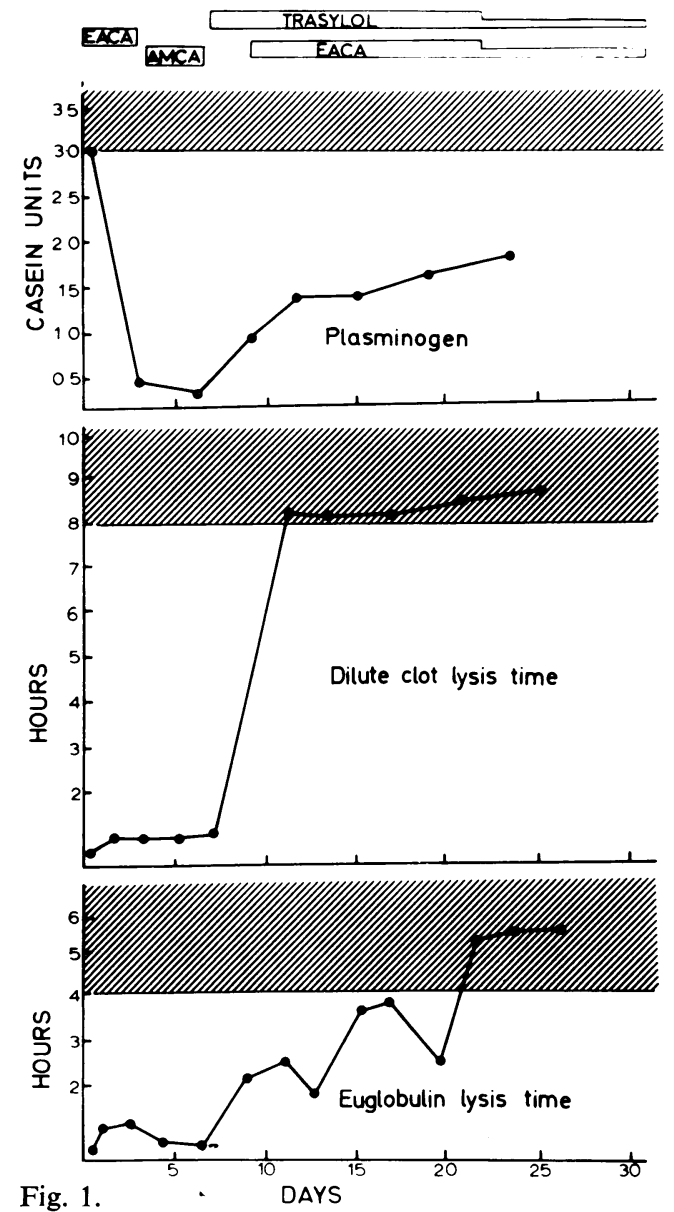

Fig. 1.

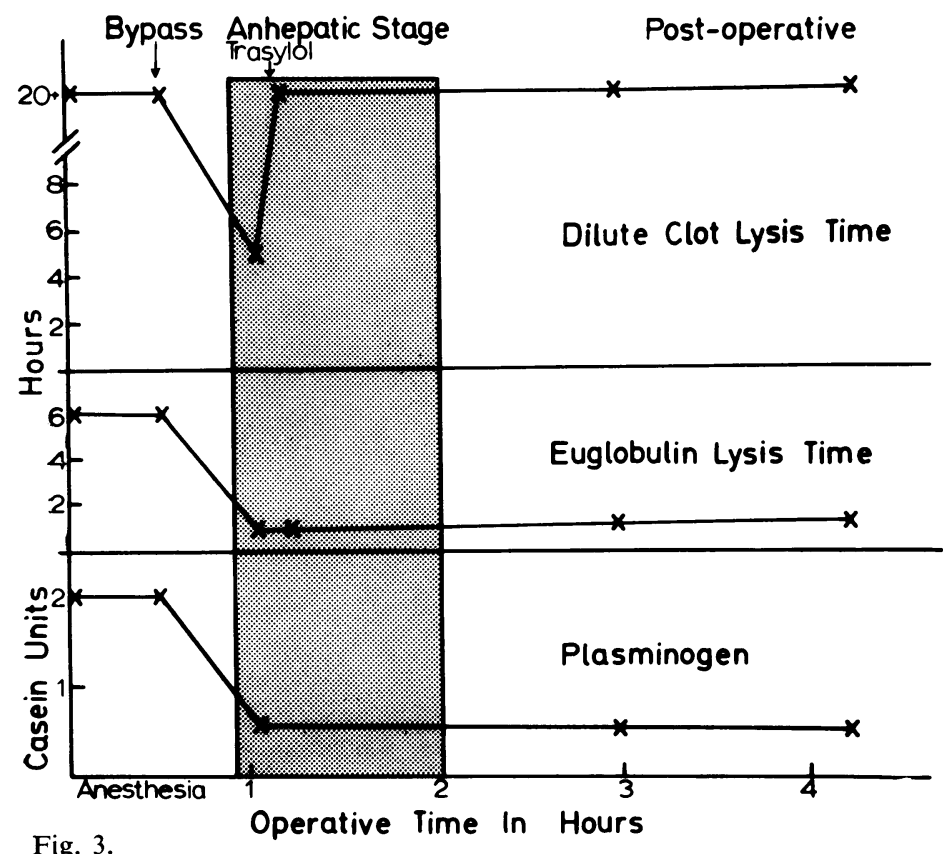

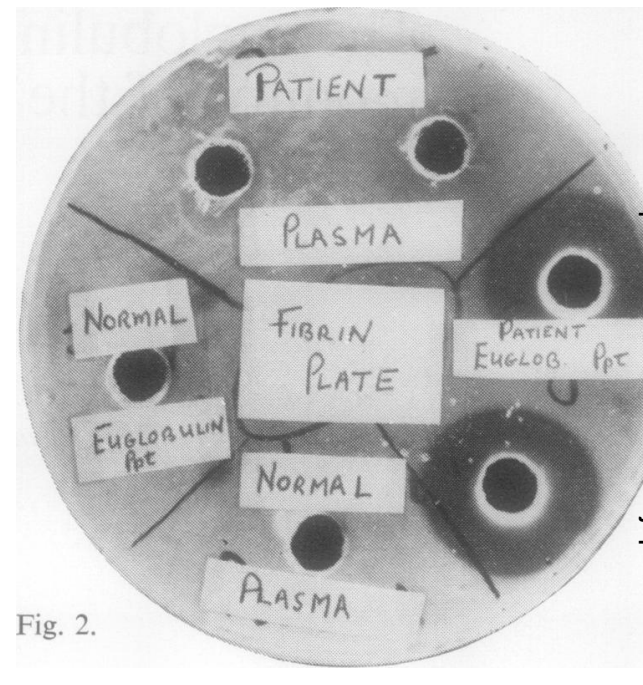

Fig. 1 Tests of fibrinolysis in a patient with acute $\mathbb{D}$ promyelocytic leukaemia receiving therapy with EACA and Trasylol. The hatched areas represent the normal range.

Fig. 2 Fibrin plate with plasmas and euglobulin fractions from a patient with acute promyelocytic leukaemia receiving therapy with EACA and Trasylo and from a normal control. Excessive fibrinolysis is 0 present in the patient's euglobulin fraction only.

Fig. 3 Tests of fibrinolysis during a liver xenograf transplant.

(Fearnley, 1960), plasminogen (Remmert a雲 Cohen, 1949), and fibrinogen (Ellis and Stranskik, 1961) assays were performed according to stand存d methods. Fibrin plates for determining fibririolytic activity were prepared according to method of Astrup and Müllertz (1952) \&s modified by Holström (1965).

\section{AMINO-ACID CHROMATOGRAPHY FOR} EACA LOCALIZATION

Chromatography was performed using Whatmân no. 1 paper. Volumes of $0.02 \mathrm{ml}$ of standard athd sample were spotted onto the paper. The sp\&ss were dried in an air current, and the chromategrams then developed by ascending chromatography. The solvent used was a butanol-acesfic acid-water solvent (butanol $120 \mathrm{ml}$, glacial acesic acid $30 \mathrm{ml}$, distilled water $50 \mathrm{ml}$ ). After 12 houtes the chromatograms were removed and dried iröa stream of air. The chromatograms were stained with ninhydrin and then placed in an oven 1 t $60^{\circ}$ to $80^{\circ} \mathrm{C}$ for five to 10 minutes to accelergite the staining process. Amino-acid sites appearedtas purplish-brown spots, and the EACA vors identified from the site of the standard. 
The euglobulin lysis time test: An ineffectual monitor of the therapeutic inhibition of fibrinolysis

Streptokinase $(\mu / \mathrm{ml})$

EACA (mg/ml)

Dilute clot lysis time (hr)

Euglobulin lysis time (hr)

$\begin{array}{rrrrrrrrrrrr}0 & 50 & 50 & 50 & 50 & 50 & 50 & 50 & 50 & 50 & 50 & 50 \\ 0 & 0 & 1 & 5 & 10 & 15 & 20 & 25 & 30 & 40 & 50 & 60 \\ >24 & 3 \frac{1}{2} & 18 & >24 & >24 & >24 & >24 & >24 & >24 & >24 & >24 & >24 \\ 6 & \frac{1}{2} & \frac{1}{2} & \frac{1}{2} & \frac{1}{2} & \frac{1}{2} & \frac{1}{2} & \frac{1}{2} & \frac{1}{2} & \frac{1}{2} & \frac{1}{2} & 2\end{array}$

Table I Dilute clot and euglobulin lysis times following the addition of increasing quantities of EACA

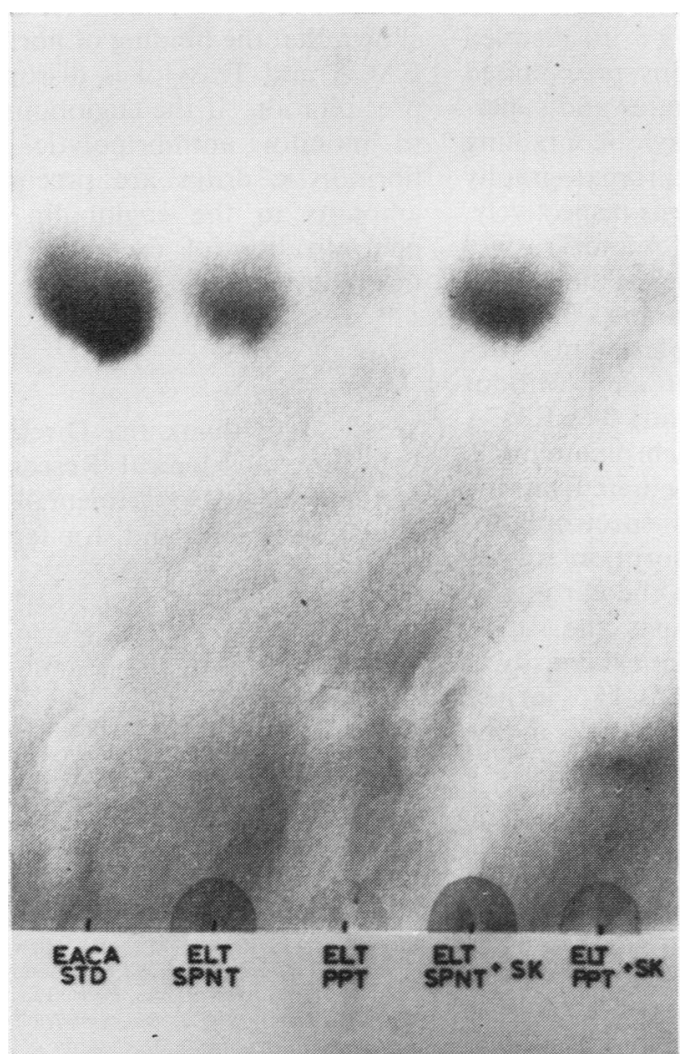

Fig. 4 Paper chromatography of fractions, as prepared for the euglobulin lysis time test, from plasma to which EACA has been added.

$E A C A S T D=E A C A$ standard; ELT SPNT = supernatant; ELT PPT = euglobulin precipitate; $E L T S P N T+S K=$ supernatant from streptokinase-activated plasma; $E L T P P T+S K=$ euglobulin precipitate from streptokinase-activated plasma.

\section{SUPERNATANT}

PRECIPITATE

TRASYLOL STD.
CELLULOSE ACETATE ELECTROPHORESIS FOR TRASYLOL LOCALIZATION In view of the polypeptide nature of Trasylol, $\frac{\bar{\sigma}}{\widehat{D}}$ electrophoresis was more suitable for identification $\cong$ than was chromatography. Electrophoresis was performed on cellulose acetate strips in a Tris- $\overrightarrow{0}$ EDTA-borate buffer, $p \mathbf{H}$ 8.8. Test samples$(0.02 \mathrm{ml})$ were run at $10 \mathrm{~m}$ amps for one hour and $\vec{\omega}$ the electrophoretic bands identified with Ponceau? $\mathrm{S}$ stain.

\section{Results}

CLINICAL SITUATIONS WITH INCREASED FIBRINOL YSIS

Hyperfibrinolysis in the patient with acute promyelocytic leukaemia was treated with a combination of EACA $24 \mathrm{~g}$ and Trasylol 1,000,000. units daily by intravenous injection. This therapy controlled the clinical bleeding and excessive fibrinolysis as evidenced by lengthening of the dilute clot lysis time, absence of fibrin plate lysis, and rise in the plasma levels of fibrinogen and $\mathbb{Q}$ plasminogen. The euglobulin lysis time, however, $\underset{\vec{F}}{\overrightarrow{2}}$ remained shortened (Fig. 1). Fibrin plate studies? showed no fibrinolysis when whole plasma was plated, but markedly excessive fibrinolysis when the euglobulin fraction only was plated (Fig. 2) In the same way, the dilute clot, but not the euglobulin, lysis times, reflected clinical control of fibrinolysis by Trasylol in the anhepatic baboon (Fig. 3).

EUGLOBULIN LYSIS TIME AND INDUCED INCREASED FIBRINOLYSIS in vitro Streptokinase $^{1}$ added to normal plasma in a concentration of 50 units per $\mathrm{ml}$ results in activation of fibrinolysis with marked shortening స్ట of the dilute clot and euglobulin lysis times. On the subsequent addition of increasing concentrations of $\mathrm{EACA}^{2}$ (1-60 mg per $\mathrm{ml}$ ) to aliquots of the streptokinase-activated plasma the dilute clot@ lysis time reverted to normal. However, the euglobulin lysis time of the activated plasma $\overline{0}$ remained abnormally short in spite of the addition $\overrightarrow{\mathbb{Q}}$ of EACA (Table).

Fig. 5 Cellulose-acetate electrophoresis of the fractions, as prepared for the euglobulin lysis time test, from plasma to which Trasylol has been added.
${ }^{1}$ Lederle Laboratories, USA ${ }^{2} \mathrm{Kabi}$, Sweden. 
DISTRIBUTION OF EACA AND TRASYLOL IN THE EUGLOBULIN PRECIPITATE AND

SUPERNATANT

To aliquots of normal plasma, and normal plasma in which the fibrinolytic activity was activated by the addition of streptokinase ( 25 units per $\mathrm{ml})$, EACA ( $5 \mathrm{mg}$ per ml) or $\operatorname{Trasylol}^{1}$ (75,000 units per $\mathrm{ml}$ ) was added. Aliquots of these plasmas $(0.5 \mathrm{ml})$ were then diluted with $9.5 \mathrm{ml}$ distilled water and the euglobulin fractions precipitated with $1 \%$ acetic acid. The precipitates and supernatants from the EACA and Trasylol-containing plasmas were submitted to paper chromatography and cellulose acetate electrophoresis respectively. The results indicated that the EACA and Trasylol were present almost exclusively in the supernatants (Figs. 4 and 5). Control samples included euglobulin precipitates and supernatants prepared from normal plasma with and without added streptokinase ( 25 units per $\mathrm{ml}$ ). The EACA and appropriate blanks on the chromatograms were eluted with $0.01 \mathrm{NHCl}$. An eluate from the EACA standard was scanned spectrophotometrically and its maximum absorption was at $250 \mathrm{~m} \mu$. The EACA eluted from the chromatograms of the euglobulin precipitates and supernatants were quantitated by the optical density at $250 \mathrm{~m} \mu$. The results indicated that $15 \%$ of the EACA was in the precipitate and $85 \%$ in the supernatant.

\section{Discussion}

The rationale for precipitating the euglobulin of plasma before measuring fibrinolytic activity is the separation of the precipitable fibrinolytic enzymes from the non-precipitable inhibitors, antiplasmin and anti-activator. Euglobulin contains the plasminogen activator and plasminogen of plasma, $25 \%$ of the fibrinogen, but only traces of the antiplasmins (Kowalski, Kopeć, and Niewiarowski, 1959). The sensitivity of this test system becomes greater than that of the dilute clot lysis time in which the fibrinolytic inhibitors are not removed. This is reflected by shortening of the clot lysis time of normal plasma from 12 to 24 hours in the dilute clot lysis time to five to 10 hours in the euglobulin lysis time.

\footnotetext{
'Bayer, Germany.
}

Sherry, Fletcher, Alkjaersig, and Sawyer (19 have shown that administered EACA is unlikety to be protein bound, for the drug is reafly dialyzable from plasma. By ultrafiltration tee niques and protein-free filtrate assays, McNicel, Fletcher, Alkjaersig, and Sherry (1962) foưhd recovery of the drug from the plasma to \$e quantitative. The present studies in addition hase shown that the binding of fibrinolytic enzymes $\frac{\mathscr{q}}{\mathrm{y}} \mathrm{y}$ EACA and Trasylol is disrupted by euglobu皮 precipitation. If the euglobulin lysis time is used to monitor antifibrinolytic therapy the antifibrinolytic drugs are precipitated in mininial amounts in the euglobulin fraction, and the neutralization of excessive fibrinolysis is thas not reflected.

We wish to thank the Director, South African Institute for Medical Research, and Professor J. A. Myburgh, Department of Surgery,University of the Witwatersrand, for facilities to carry abit these studies.

References estimating fibrinolytic activity. Arch. Biochem., 40, 346-351.

Ellis, B. C., and Stransky, A. (1961). A quick and accurate methd for the determination of fibrinogen in plasma. J.Lab. $\mathrm{Q}$. Med., 58, 477-488.

Fearnley, G. R. (1960). Spontaneous fibrinolysis. Amer. Cardiol., 6, 371-377.

Holmström, B. (1965). Streptokinase assay on large agar diffus plates. Acta chem. scand., 19, 1549-1554.

Kowalski, E., Kopeć, M., and Niewiarowski, S. (1959). An evaluation of the euglobulin method for the determinatin of fibrinolysis. J. clin. Path., 12, 215-218.

MacKay, D. G. (1965). Disseminated Intravascular Coagulation: An Intermediary Mechanism of Disease. New York ald London, Harper Row.

McNicol, G. P., Fletcher, A. P., Alkjaersig, N., and Sherry (1962). The absorption, distribution and excretion $8 \mathrm{f}$ $\epsilon$-aminocaproic acid following oral or intravengus administration to man. J. Lab. clin. Med., 59, 15-24.

Merskey, C., Johnson, A. J., Kleiner, G. J., and Wohl, H. (1967). The defibrination syndrome: clinical features and labogatory diagnosis. Brit. J. Haemat., 13, 528-549.

Nilsson, I. M., and Olow, B. (1962). Fibrinolysis induced streptokinase in man. Acta chir. scand, 123, 247-266.

Pechet, L., Groth, C. G., and Daloze, P. M. (1969). Changes coagulation and fibrinolysis after orthotopic canine liver homotransplantation. J. Lab. clin. Med., 73, 91-102.

Remmert, L. F., and Cohen, P. P. (1949). Partial purification and properties of a proteolytic enzyme of human serung. J. biol. Chem., 181, 431-448.

Sherry, S., Fletcher, A. P., Alkjaersig, N., and Sawyer, W. $\mathbb{D}$. (1959). E-Aminocaproic acid. 'A potent anti-fibrinoly? agent'. Trans. Ass. Amer. Phycns., 72, 62-70. 